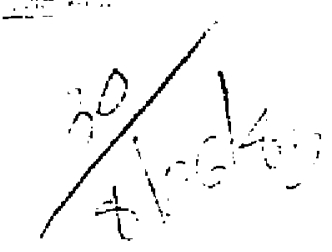

$$
I-22628
$$

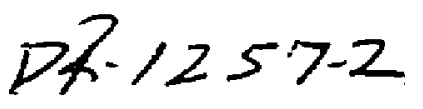

SLAC - PUB -3735

July 1985

(M)

\title{
LIMITING TECHNOLOGIES FOR PARTICLE BEAMS AND HIGH ENERGY PHYSICS *
}

\author{
Wolfgang K.H. Panofsky \\ Stanfard Linear Accelerator Center \\ Stanford University, Stanford, California, 04905
}

SLAC-PUB --3735

DE85 017003

Presented at the 1985 SLAC Summer School on the Physics of High Energy Particle Accelerators, Stanford, California, July 15-26, 1985

* Work supported by the Department of Energy, contract DE - ACO3-76SF00515. 
Since 1930 the energy of accelerators had grown by an order of magnitude roughly every 7 years. Like all exponential growths, be they human population, the size of computers, or anything else, this eventually will have to come to an end. When will this happen to the growth of the energy of pasticle accelerators and colliders? Fortunately, as the energy of accelerators has grown the cost per unit energy has decreased almost as last as has the increase in energy. The result is that while the energy has increased so dramatically the cost per ncw installation has increased only by roughly an order of magnitude since the 1930's (corrected for inllation!), while the number of accelerators operating at the fronties of the field has shrunir. As is shown in the by now familiar Livingston chart (Fig. 1) this dramatic decrease in cost has been achieved largety by a succession of new technologies, in addition to the more moderate gains in efficiency due to improved design, economies of scale, etc. We are therefore facing two questions: ( $I$ ) It there good reason scientifically to maintail the exponential growtb?, and (2) Are there new technologies in sight which promise continued decreases in unit costs? The answer to the first question is definitely yes; the answer to the second question is maybe.

Preceding speakers today have outlined the reason why collision energies in the $1 \mathrm{TeV}$ range, measured in the center-of-mass frame of the fundamental constituents (quarks and gluans) should be a fertile region. Although high energy physics hes scored spectacular successes during the last decate in simplifying our uлderstajding of matter, we are facing the not uncommon consequence that as understarding increases new questions open up. Although the "standard model" unifying the weak and electromagnetic interactions has been an ungualified success in terms of agreement with experiment, it has many unsatisfactory features. There are some 20 arbitrary parameters in the theory. Unification of the theory with the strong interaction is still incomplete. We have discovered three families of quarks and leptons which differ only in mass but appajently not in other fusdamental properties. What defines the masses? Will ther: be further generations or is this aesthetically appealing picture the end? $W=$ do not k110w. In 
particular we do not understand the mechaniam which diferentiates the masses of the carriers of the various interactions. Although three families of quarks and leptons seem to be a nice aimple number if there were no further generations, the total number of so-called elementary constituents is atill disturbingly large if one counts all of them. In fact if one adds un the 18 colored quarka, 6 leptons, the photon, the 3 massive vector bosons which carry the electro-weal force of the standard model, and the 8 gluons which are the transmitters of the strong intoraction, together with perhsps 1 graviton which carries the gravitational force, then we obtain a total of 37. This is a lot of particles to be "eli 'nentary."

The $1 \mathrm{TeV}$ region for the center-of-masg energy of collisions among fundamental constituents is apt to provide the answer to some but not all of these questions and it surely will open up eome new ones again. In particular, work in this energy region is likely to sort out the various alternate models conjectured by theorists for the otherwise unexplained mass differences amoing sorne of these particles, or it may uncover other phenomena or explanations which are rot on the conjectured list.

Accelerator builders face a índamental dilemma. Protons are ccmposite particles, while electrons dow, to dimensions of $10^{-16} \mathrm{~cm}$ ere still point-like. As a result, the energy of collisions among protons is shared among the quarks and gluons. Therefore, depending on the reaction under atudy, the energy of procons has to be "derated" by about an order of magnitude relative to electrons in providing the same "reach" into the unknown. Thus electron accelarators of considarably lower energy are equivalent to the corresponding proton acceleerators. On the ather haud, electron accelerators at ultra-high energies using bnown technologies are becoming excessively expensive. But this is not all there is to the electron-proton comparison. The total cross-5ection in proton-proton collisions is still on the increase, while the cross-section for producing events extibiting nzw physical phenomena of it terest is expected to decrease as the ar rare of the relevant mass range to be investigated. Therefore, the rigud-to soise ratio for proton-proton colliders for jnteresting ever ts is degenerating rapidly with 
energy, while the total event rate continues to increase. As a result the data analysis process applicable to hadron-hadron collider events is a frightening process which has to reject, hopefully successfully, a substantial nomber of supposedly unneeded features of the primary collision. While this can be accomplished with confidence in designing experiments with reasonably wall-defined objectives, the lingering doubt ramains that the increasing need for rejecting primary data might also reject unarticipated primary discoveries. Therefore, given the choice, most particle physicists would prefer an electron-positron collider of oute-tenth of the particle energy to a corresponding proton-proton collider of roughly comparable luminosity. But does this choice exist? The arr e: that in the TeV range it does not within present technojogies.

The SSC is a boid step forward in extending the proton-proton collider technologies using superconducting toagnets ćemonstrated so successfully at Fermilab into the multi-TeV energy range meas ured in terms of collision energy of the fundamental cunstituents. This proposal does not involve basically new technology tur hopes to extend the energy range of the decrcasing unit cost by economies of scale and simply good design. Corresponding matching opportunities for electrons do not exist today.

The reason for this conclusion is known to all of you. Electrons in storage rings emit synchrotron radiaticy and the rate of energy loss per torn increases as the fourth power of the cnergy. The cost of storage rings in essence is composed of two terms: (1) proportional to the sadius or physical size of the device, and (2) proportional to the fourth power of the energy divided by the orbit radius. If these two tarms are matched a quadratic scaling law of cust vo. energy casults. Thus it is likely that LEP with a circumference of 27 kilometers will be the last electron-positron collider bullt along the by-now estab'ished storage ring pattern.

For the above reasons it is evidest that if the exponential growth in energy for electron collisions and for proton collisions beyond the SSS is to be maintained new technology is needed. We are thus waiting again for the pioneers to devise 
these new techrologies just as the new developments invented by those people we are honoring tomorrow have advanced the accelerator frontiers and have made. it possible to maintain the exponential growth. One of the hopefully succersful missions of this smmmer school is to create among the younger generation those who will reduce this expectation into practice.

These are optimistic remarks but even the most inventive people in this audience or elsewhere must in the future deal with certain fundamental limits. If we are to extend electron-positron collisions to higher energies we must abandon storage riugs, unless someone makes an invention to suppress synchrotron radiation. Thus the electron-positron collider of the future must be a linear collider in which two linear machines accelerating electrong and positrons are aimed at one another to produce the required rate of interaction.

Such linear colliders lose the primary acivantage of storage rings which is the Fact that the same electrons and positrons collide repeatedly before eventually being lost due to the finite lifetime of the stored beam. In consequence, as we shall see, useful designs for linear colliders require that both the total beam power required to achieve adequate reaction rates and the density of the particle beams during interaction must become very large.

The reason for this conclusion derives from the unpleasant fact that the total energy of each beam will have to be thrown away after each collision between opposing bunches during which only an exceedingly smell fraction of electrons and positrons prodace events of $i$-terest. The only exception to this situation would arise if energy recovery of the "spent beam" would somehow be possible. This latter possibility has been explored by severa! physicists, in partictlur $U$. Amaldi, by uring the epent beam to produce radiofrequency energy in a sup arconducting linear accelerator; that accelerator thug energized can then be used for accelerating the mext Eunch. Elejuentary arguments make it clear that there is hope for wuch energy recovery only if 4 values of supersonducting acceler.tors can be attuined which are two orders of magnitude or 80 larger than those o w 


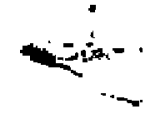

deemed feasible. Current linear acceleratora at room temperature are operating at $Q$ values of the order of $10^{4}$ and use duty cycles of the order of $10^{-5}$. Thus if a guperconducting accelerator running continuously is to utilize refrigeration power comparable to the if power fed into conventional accelerators, then we requira $Q$ values of $10^{4} \times 10^{5} \times 10^{3}=10^{12}$, where the last factor of $10^{3}$ is the reciprocal of the efficiency of refrigeration at temperalures usable for auperconducting devices. The $Q$ value of $10^{12}$ is about $10^{2}$ to $10^{\mathrm{J}}$ larger than practical valtes attained to date. In additjon, energy secovery in superconducting accelerators has numerous other practical problems which may or may not be more severe than those faced by other devices at room temperature which we will now discuss.

If encrgy recovery is provert infeasible, then we have to face the power consumption inherent in throwing the interacting bunches away after collisions. We can write an equation which directly relates the luminosity $L$ of interaction as related to the frequency $f$ of collision, and the cross section $\boldsymbol{A}$ of the interacting beams:

$$
L=\int N^{2} / A
$$

where $\mathrm{N}$ is the number of particles per bunch. If we assume that the beam is circular in cross-section and has an invariant emittance $\epsilon_{m}$, and if the opposing bunches are focused into collision with a focusing parameter $\boldsymbol{\beta}$, then the average power $P_{B}$ of each of the colliding beams is given by:

$$
P_{B}=m_{0} f N_{\gamma} \quad \text { and } \quad L=f N \gamma^{2} / 4 \pi \epsilon_{n} g
$$

The number of particles which we can handle per bugch is limited by the energy spread produced due to the beamsstrahlung of the electrons or positrons during collision. The very factore, in particular the density of interaction, which eshance luminosity alno increase the electromagnetic radiation experienced by an opposing particle. One might assums that this beamsstrahlong can be calculated by the applicable classical forumlas. However, using the ordinary expressions for 
sychrotron tadiation, we find that the trequency apectrum of the emitteri radiatlon extends up to the cribe of the cyclotron frejuency of the emitting particle in tho electromagnatic feld of the opposing bunch; in otiuer words, the opper limit of the esergy spectruen in general growu faster than the ene $2 y$ of the colliding partieles themselves. Once the top enercy of the radiated photons exceeds that of the primary buan the clansical calculation fo no longer valid; as a practical matter the spectrum is eut off at an egergy equal to thst of the primas beam. If wo asume that the parameters of an electron-positron collider are of interest for the aext generation machines only If such a quantum mechanical cutoff in the oynehrotron radiation opectrum in required, then we can write formulas relating the parametert of interest to the physicists to the parameters which the machine buildar would consider limiting in design.

The physicist has to specify three basic parameters;

(a) the required beam energy

(b) the luminosity

(c) the tolerable energy spread $\delta_{q}$ produced in the interaction.

The latter is partieulariy important should there be resonances in the new energy range to be investigated. This importance is somewhat moderated by the fact that if there are resonances the cross sections would be larger at such a racopance and one could therefore afford to decrease the luminosity at the rasonance in order to achieve a narrower energy width. With the exception of this unlikely situation reasonably broad energy spreads - say in the 50-30\% jange - could be tolerable. However it is unavoidable that within current quantummechanical wisdom the requited luminositjes would have to go up with the square of the enorgy in order to give reasonable rates for events of interest. A luminosity

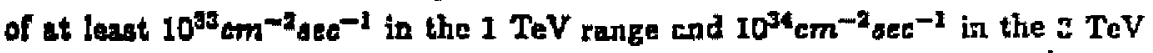
range appear essential if event rates of interest of the order of $10^{2}$ to $10^{3}$ per year are to be anticipatsd. 
In addition to these preliminary experimental parametere thete we also secondary requirements of concern to the phyaicists. Backgtound due to beam ges interactione or radiation from upstream or downstream foescing devices must be held at tolerable llmits. From the point of view of event raeonstruction one would like to limit severely the expected number of events pex bunch crossing. In addition, one would like to make it possible to place deteetors very close to the interaction point in order en be able to detect short-lived decays. If posslble the accelerating mechanism should preserve polarization so that events orfginating from states of specified angular momentum can be isolated. Bowever, fo these considerations let me assume that the primary factor, luminosity, eatigy and energy width are all that define the usefulness of a machine.

A problem is that at present it is not fully clear which machine parameters ara controlling the economics of the overall machine. Average benm powar combined with the finite efficiency of generating the beam in clearly a very important and probably the most important parameter. In other worde, linear collider at super high energy must operate heavily loaded.

Let me make a remark on the somewhat arcane "voodoo" economics in which the accelerator physicist defines the construction cost for a machine. In the past the government has been persuaded to supply appropriate afequete construction funds to build a new facility. With the exception of inflution all such monies are directly applied to the selevart construction cost. In contrast, when a public vtility builds a power plant the money needed may be as large as threa times the actual cost of construction due to the cost of raising the aecessary capital and the accumulating interest rate during the construction period. Therefore, considering the large power probably required for aceslezator of the future, one might even entertain the notion that a power plant might be part of the projected construction cost. This, of course, is not a saving in real etonomit terms.

The attainable accelerating gradient and therefore the cepromically (or po- 
litically!) practical length of a machine may or may not be a constraining factor. Because of the high power costs the efficiency of extracting the stored energy in the accelerating dcevices by the beam is importent. Since the total amount of energy per bunch is limited by the energy spread produced in the beam-beam interaction attainment of such extraction efficiency can become more dificult if the accelerating gradients are very large and therefore the energy stored in the accelerating structure is correspondingly increased. Therefore the shortest accelerator is not necessarily the most economical accelerator overall, although it is obviously much more attractive aesthetically and reduces the total impact of the installation.

Having said all this we can write a number of basic equations which define the various derived quantities for accelerator design in terms of the specified physical parameters. Since in the muIti-TeV region the quantum mecharical cutoff of the syachrotron radiation spectrum will enter into the picture I am giving these equations only in that region.

$$
\begin{aligned}
& N=\frac{4 \pi}{(1.63)^{3} \alpha^{4}}\left(\frac{\delta_{q}^{3}}{D}\right) \\
& A=\frac{4 \pi}{(1.63)^{3} \alpha^{4}}\left(\frac{P_{b} \delta_{q}^{3}}{E \bar{D}}\right) \\
& f=\frac{(1,63)^{3} \alpha^{4}}{4 \pi}\left(\frac{P_{b} D}{E \overline{\delta_{q}^{3}}}\right) \\
& \sigma_{x}=\frac{1}{4 \pi}\left(\frac{P_{b} D}{r_{\mathrm{e}} m_{e} c^{2} L}\right)
\end{aligned}
$$

In these equations $\mathrm{D}$ is the "disruption" parameter which is the ratio of the bunch length at interaction to the focal length produced by the electromagnetic focusing effect in the beam-beam interaction. This number cannot be very large because if it were each particle would undergo oscillations within the bunch of the 
opposing parlicle, and eventually these oscillations would become unstable. For moderate values of $D$ this mutual focusing effect is beneficial in that it increases the average beam density and therefore the luminosity.

The above equations are written for beams of circular cross sections. Additional advantages ca:: be abtaised using "flat beang" because then for a given bearn density the total disruptive beam-beam interaction and the beamsstrahlung effects are reduced. If for the time being we ignore this possibility and also ignore the enhancement produced by the beam-beam interaction (which at most can become a factor of 6), we can combine the aboye equations by eliminating the $D$ factor and write a simple expression for the beam power as follows:

$$
P_{b}=5\left(\frac{L}{10^{30}}\right)\left[\frac{E_{n} \beta o_{z}}{\delta_{q}^{3}}\right]^{1 / 2}
$$

Here the power is measured in megawat ts and fominosity in $\mathrm{cm}^{-2} \mathrm{sec}^{-1}$. The quantity $\varepsilon_{n}$ is the invariant emitance, that is $r r r^{\prime}$, and $\beta$ is the usual focusing parameter and $\sigma_{F}$ is the bunch length, all mengured in centimeters. Note that the required luminosity would have to increase as the square of the energy.

Nole that this equation is totally independent of the means which are employed to produce beams and bring them into collision. Such acceleration would be achieved by traditional if structures or some new, for instance laser, devices.

It is interesting to plug numbers into this equation under the assunption that accelerator performance was no belter than that employed in the design of the SLAC SLC, but assuming that we would be producing $5 \mathrm{TeV}$ per beam, rather than $50 \mathrm{GEV}$ per beam os is the case for the SLC. If we assume that the luminosity is to scale by the square of the energy from the design luminosity of $6 \times 10^{30} \mathrm{~cm}^{-2} \mathrm{sec}^{-1}$ of the SLC, the invariant emiltance $\varepsilon_{\mathrm{n}}$ is $3 \times 10^{-3} \mathrm{~cm}$, and the 6 vilue $1 \mathrm{~cm}$, and if we assutue a permiscible energy spread $\delta_{q}$ of $30 \%$, then wo require a beam power well in excess of $10^{4}$ Megawats. This is clearly impractical and therefore one has to go considerably beyond SLC paraneters if the linear 
colfider idea without energy recovery is to become practical in the TeV rango. Since the SLC is conidered to bo a "daring" machine by many, ouch a further extrapolation is clearly not an immediate prospect and thus the time scale at which the SSC (whteh is based on exirting technology) and ouch a super linear collider can be achieved are many years apart.

In order to go beyond the SLC performance the following steps come to mind:

1. make the beam non-circular,

2. reduce the normalized (invariant) emittance,

3. reduce the bunch length.

4. reduce the $\beta$ value in the interaction region.

A non-circular beam with aspect ratio $R$ in principle introduces a factor $2 /(1+R)$ into the power equation. This is not a very steep dependence and there ara limits to the practicality of focusing an asymmetric beam to the required small dimensions considering the extreme complexity of the final focus system when designed to hapdle beams of finite energy spread.

Reducing the normalized emittance $\epsilon_{n}$ is in practice a matter of designing - damping ring to "cool" the radial temperature of the electrons and positrons to an extent greater than that projected for the SLC. Increaging the brightness of the primary gun is not of major relevance to achieve reduction of the normalized emittance since the phasc volume of the positrons is determined by the electromagnetic shower process in the positron converter and not by the driving beam.

The ultimate performance of damping rings has not been thoroughly studied but an improvement in $c_{n}$ by perhaps 2 orders of magnitude appears feasible. One thould note, however, that if the particle energy is increased beyond the SLC and if the normalized emittance is decreased, then both of these factors decrease the actual beam djameters at the interaction point below the $1.2 \mathrm{mi}-$ crameters now prajected for the SLC. If we reduce the normalized emittance by 
2 orders of magnitude and increasc energy by 2 ordern of magnitude, then the beam diameter is decreased by about 100 and the beam area by a factor of $10^{4}$. We are talking about beams of radium of perhapa 10 angotrom units. Although beams of such dimensions have proven practical in scanning electron microscopes, the question how to design final focusing systems with sufficient freecom from aberrations, sufficient focusing strength, and mechanical stability has not even been considered.

The longitudinal dimension $\sigma_{s}$ could also be shrunk frod the 1 millimeter value of the SLC, let w say lo 1 micron. All these factors combined would bring the average beam power into the few megawatt rasige. This might, perhaps, permit a practical design. provided the efficiency from the wall plug to the bearn is not too small. Note, however, that the beam density would become of the ordar of $10^{27}$ electrons $/ \mathrm{cm}^{3}$ (!) very much targer than ordinary macroseopic densities.

A similar problem arises in respect to the a:celerating structure. If we assume that a damping ring can produce normalized emittances well below those projected for the SLC we are facing an increased problem of growth of this emittance in the actua! accelerating and $6 \mathrm{nal}$ focusing structures.

Many different kinds of accelerating structures and methods bav. been pro. posed and are under intensive study. Time coes not permit me to discuss these here. Some of these are plasma devices in which a laser beam or laser beams interfering with one another produce waves in a plasma which, in turn, accelerate the particles. Such devices appear attractive because of the potentially high gradient they might produce. However, 1 am personally pessimistic about their utility in this case because of two factors: (1) the overall efficiency of transfer of power source to the beam, and (2) the funclamental dificulty in coatrolling the exact micro-detail of the plasma to avoid growth in the emittarre of tie beam. Other accelerating metheds use either the electric fields of lasers or microwave energy to produce acceleration.

The most predictable performance would involve "conventional" if struetures 
which, however, must be fed by power sources not as yet developed. All such cources are in effect tranformers from low voltage, high current devices to the high energy, low current bearn to be produced for collision purposes. The "convantional" electron linae uses a multiplicity of such high current beams in the klystron tubes feeding the machine. The most challenging of such transformers are in essence two beam devices. In one design a hollow beam of high intensity is transmitted at the edge of a conventional if structure where the magnetic field is large and the electric field is small. The beam to be accelerated passes at the axis where the electric feld is bigh and the magnetic feld is zero. Iis another version a many kilo-ampere beam is produced in an induction accelerator and If energy is extracted periodically either by a free electron laser, that is by use of a wiggler, or with conventional if cavities if the primary high current beam is bunched. The energy loss corresponding to the extracted of is replenished by re-acceleration with induction cores. The mierowave power is then fed from the extraction points to the high energy accelerator. Such two beam devices have an inherently high efficiency.

The problem with all such devices deals with wake field effects, in particuler in respect to transverse deflecting modes. Any medium which supports the required longitudinal arcelerating fields will also support transverse modes, and therelore the required alignment tolerances will become extraordinarily severe if, as indicated above, emittances much smaller than those projected for the SLC are to be employed. Sinte the optimization of parameters suitable for this purpose points to wave length much shorter than the customary S-band range, this situntion would be aggravated.

Reducing the $\beta$ value below $1 \mathrm{~cm}$, or as far as that goes even to attain $1 \mathrm{~cm}$ for beam energies as high as $5 \mathrm{TeV}$, is an unsolved problem. An exciting possibility is to employ 2 bunches, one to produce the focusing field to contract the second bunch which is the one to yield the desired collision intensities. Such first arder lenses produced by placing charge into the beam are potentially a great deal more powerful thas existing external focusing lenses such es superconducting 


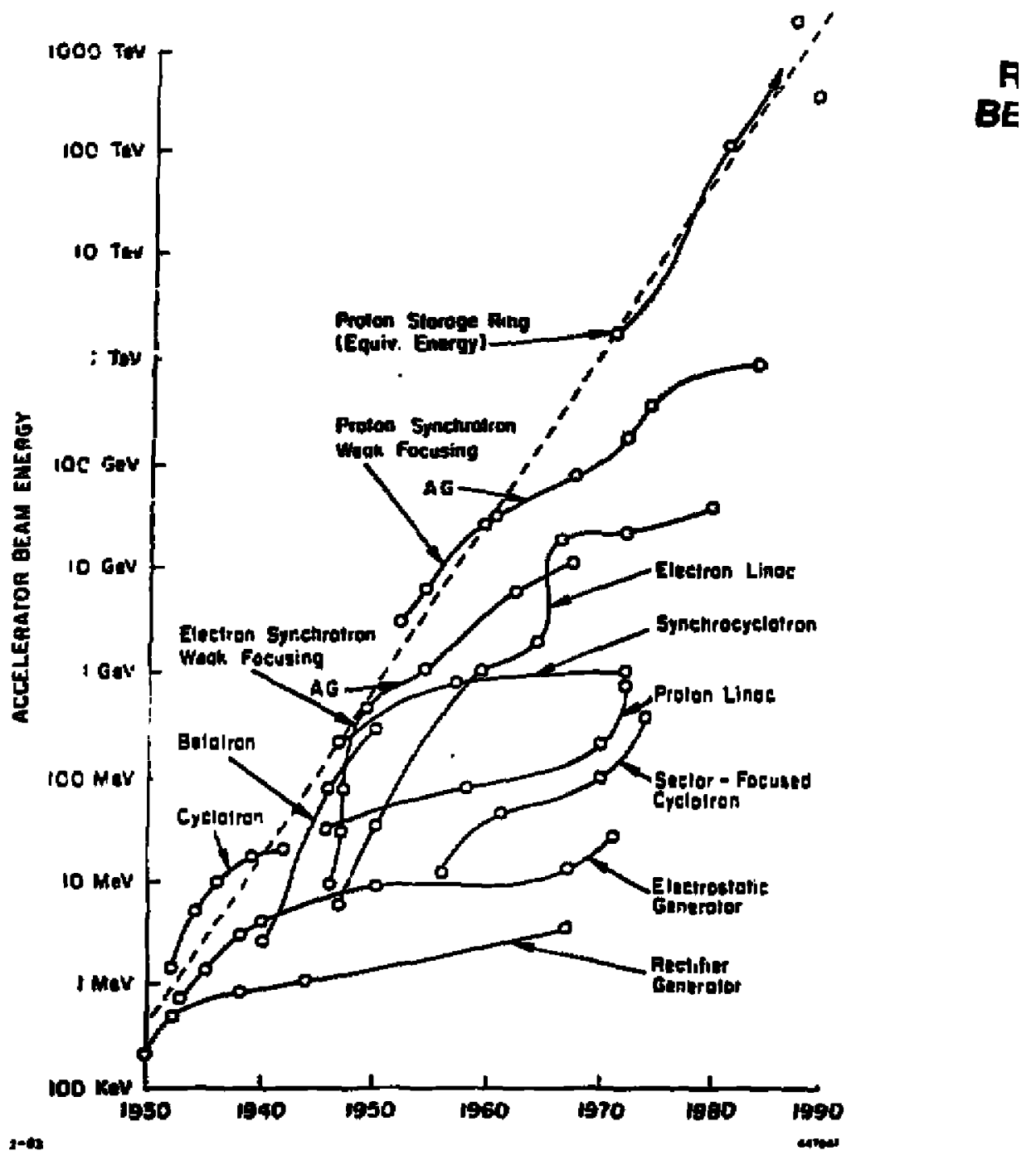

Fig. I 